\title{
The Effect of Coalescence Behavior of Liquid on Bubble Column Hydrodynamics
}

\author{
Mojtaba Goraki Fard, Youssef Stiriba, Xavier F. Grau \\ Departament d'Enginyeria Mecanica, Universitat Rovira i Virgili \\ Av. Paisos Catalans 26, 43007, Tarragona, Spain \\ mojtabagoraki.fard@urv.cat; youssef.stiriba@urv.cat; francescxavier.grau@urv.cat
}

\begin{abstract}
In this work, Finite Volume Method (FVM) is used to simulate the gas-liquid dispersion and the effect of alcohol addition on the gas hold-up, liquid circulation velocity and gas velocity in a bubble column using air-water system to which $0.01 \%$ concentration propanol was added. The model aims to describe the flow pattern of air-water system and the impact of surfactant addition on the local hydrodynamics in a bubble column reactor. A multiphase Euler-Euler approach with mean bubble size and a standard $k-\varepsilon$ model of turbulence are used. The 3-D Computional Fluid-Dynamics (CFD) simulations are able to predict the liquid circulation, the gas holdup and the effect of the surfactant addition on the flow pattern.
\end{abstract}

Keywords: Bubble column reactors; Finite Volume Method; Hydrodynamics; Alcohol addition

\section{Introduction}

Bubble column reactors (BCR) are widely used in the chemical and biochemical industries due to their simple geometry, easy manufacturing, low energy consumption, easy design/scaling up and shear stress [1], [2] and [5]. In these sorts of multiphase reactors, gas phase is dispersed in the form of small bubbles in the liquid phase. The hydrodynamics, mass transfer and chemical reactions in multiphase bubble column reactors are strongly coupled as well as the two-phase flow structure is very complex which make their design and scale-up very difficult, see [1] and [2].

A main issue of two phase flow process in bubble column reactors is, therefore, to predict accurately these aspects, which require fundamental understanding and modeling of the underlying mechanisms. The modeling of the hydrodynamics is widely affected by the physico-chemical properties of the two-phase system. In particular, bubble coalescence inhibition, usually encountered with aqueous solutions containing surfactants or industrial non-coalescence organic mixtures, and may affect the gas hold-up and the hydrodynamic regime.

The potential ofCFD for simulating hydrodynamics and gas-liquid mass transfer in the design and scale-up has been established by several authors, see for example [1-4]. The majority of previous experimental and numerical works aimed at describing the hydrodynamic parameters using air-water systems or other Newtonian and non-Newtonian liquids.For instance, in [5] the authors investigated the effect of small alcohol addition on hydrodynamics and bubble properties using different distributors. Gourich et al. [6] analyzed the mass transfer in high aspect ration column with both water and noncoalescence inhibiting liquid mixture. Buwa and Ranade [4] carried out experiments with coalescence inhibiting liquid.Besagni et al. [7] conducted some experiments to consider the effect of ethanol on the global gas holdup, flow regime and bubble distribution. Their results indicatethat near the sparger zone, there is a large number of large bubbles that break into smaller bubbles, the gas holdup is increased and the homogeneous flow is stabilized. McClure et al. [8] carried outsome experimental measurments and CFD modelling for air/water system and analyzed the impact of 2propanol addition. However, very few studies and modelstackling non-coalescence organic mixtures media, have been reported.

The purpose of this work is, therefore, to develop a computational fluid model to study hydrodynamics and the flow pattern in the air-water system and water-alcohol solutions involved in a bubble column, so that robust scale-up and performance can be achieved.Here, we present our first results using the experimental data from the works of Gourich et al. [5] to validate the CFD model. The simulationsare carried out in a cylindrical bubble column reactor of $10 \mathrm{~cm}$ internal diameter and $3 \mathrm{~m}$ height. The gas-free liquid height was about $1.66 \mathrm{~m}$. Available data describes the respective influence of 
superficial gas velocity from 0.6 to $8.7 \mathrm{~cm} / \mathrm{s}$ and of water properties on hydrodynamics using real drinking water and aqueous solutions of propanol at $0.01 \%$ concentration, ambient temperature and atmospheric pressure. The initial gas bubble size was set by experimental data. The numerical solutions for the equations model were obtained using the OpenFOAM (Field Operation and Manipulation)software package [7]. The general properties inside the flow field such as gas hold-up and local liquid velocities were calculated and compared with experimental data.

\section{Simulation Set-up}

\subsection{Two-phase flow model}

The model used in this work considers a three-dimensional gas-liquid flow with a single bubble size distribution. An Euler-Euler approach was used, where both phases (the continuous liquid phase and the dispersed gas phase) are modeled as two interpenetrating continua. The volume-averaged Navier-Stokes equations are used to describe the motion of each phase. Assuming no mas mass transfer between phases, the continuity equation for each phase is introduced as:

$$
\frac{\partial\left(\rho_{k}\right)}{\partial t}+\nabla \cdot\left(\alpha_{k} \mathbf{U}_{k}\right)=0
$$

where the subscript $k$ denotes the $k^{\text {th }}$ phase ( $L$ for the liquid and $G$ for the gas), $\rho$ is the phase density, $\alpha$ is the volume fraction, and $\mathbf{U}=(u, v, w)$ is the averaged phase velocity vector. The equations of concervation of momentum for each phase are defined as:

$$
\frac{\partial\left(\alpha_{k} \rho_{k} \mathbf{U}_{k}\right)}{\partial t}+\nabla \cdot\left(\alpha_{K} \rho_{K} \mathbf{U}_{K} \mathbf{U}_{K}\right)=-\alpha_{K} \nabla p+\alpha_{K} \rho_{K} \mathbf{g}-\nabla \cdot\left(\alpha_{K} \rho_{k} \mathbf{R}_{K}\right)+\mathbf{F}_{k}
$$

where $p$ is the pressure, $\mathbf{g}$ is the gravitational acceleration, $\mathbf{R}_{k}$ is the $k^{\text {th }}$ phase Reynolds stress tensor, and $\mathbf{F}_{k}$ represents the inter-phase momentum exchange between phase $k$ and phase $l$.The interfacial forces acting on two phases include the interphase drag, lift, virtual mass, wall lubrication, and turbulence dispersion forces. In the present study, the drag force contribution to $\mathbf{F}_{D}$ for the dispersion phase is taken to be:

$$
\mathbf{F}_{D}=\frac{3}{4} \frac{\rho_{L} \alpha_{G}}{d_{b}} C_{D}\left|\mathbf{U}_{r}\right| \mathbf{U}_{r}
$$

where $\mathbf{U}_{r}$ is the relative velocity, which is the mean velocity difference between the phases, $d_{b}$ is the equivalent diameter of the bubbles, and $C_{D}$ is the drag coefficient. A single bubble size estimated from experimental data was used. The inter-phase drag coefficient is computed using from Schiller-Naumann and Ishii-Zuber [10] assuming spherical bubbles. The lift force is introduced as:

$$
\mathbf{F}_{L}=\alpha_{G} \rho_{L} C_{L} \mathbf{U}_{r} \times\left(\nabla \times \mathbf{U}_{L}\right)
$$

where $C_{L}, \rho_{L}$ and $\alpha_{G}$ are lift coefficient, liquid density as well as gas volume of fraction respectively. The correleation of Tomiyama et al. (see [3]) is adopted in this work. The virtual mass force is indicated as:

$$
\mathbf{F}_{v m}=-\alpha_{G} \rho_{L} C_{v m}\left(\frac{D \mathbf{U}_{L}}{D t}-\frac{D \mathbf{U}_{G}}{D t}\right)
$$

Where $C_{v m}$ is the virtual mass coefficient and assumed to be 0.5 . The turbulent dispersion force is introduced as:

$$
\mathbf{F}_{T D}=\rho_{L} k_{L} C_{T D} \nabla \alpha_{G}
$$


The coefficient of the turbulent dispersion force $C_{T D}$ is assumed to be in the range of 0.1-1.0.

Flow conditions were assumed to be turbulent. Therefore, the stress tensor in the liquid phase was computed by means of the dispersed $k-\varepsilon$ turbulence model, accounting for the additional bubble-induced turbulence and gas dispersion force (see, e.g., Bannari et al., 2011). This uses the standard $k-\varepsilon$ equations for the continuous phase and includes additional source terms to incorporate effects of the dispersed phase on turbulence with the standard single-phase parameters, $C_{\mu}=$ $0.09, C_{1 \varepsilon}=1.44, C_{2 \varepsilon}=1.92, \sigma_{k}=1$, and $\sigma_{\varepsilon}=1.3$.

\subsection{Numerical discretization and boundary conditions}

The numerical solutions for the equations model in the bubble column reactor were obtained using the open source package OpenFOAM (Field Operations And Manipulations) [12]. The model described in this work is based on the existing twoPhseEulerFoam solver of version 2.2.x. The schematic view of the reactor and the corresponding computational domain used in the present 3-D CFD simulations are shown in Fig. 1.

The geometry and the grid used in the simulations were created using the Gmsh software, see Fig. 2. Very fine cells where created near the walls to solve the boundary layer. Then, the mesh sensitivity analysis was carried out using three different meshes and yielded quantitavely grid-independent results using 123700 computational cells as illustrated in Fig. 3 and 4.The boundary conditions used in the simulations are listed in Table 1. Initially, the reactor is filled with water at a height of $1.66 \mathrm{~m}$ with air above and the flow is at rest in both phases. The equations were integrated with the finite-volume approach. The convective terms of the momentum and turbulent quantities equations were descritized with the secondorder upwind scheme. The convective term of the equation fot the phase fraction were descritized with a second-order total variation diminishing (TVD) scheme. Time integration was performed with the first-order Euler scheme. All the other terms were discretized with a second-order central scheme. The calculations are started with a time step of $10^{-4} \mathrm{~s}$ for the first 1000 steps and then $10^{-3} \mathrm{~s}$ for the remaining time steps. The simulations are carried out for $50 \mathrm{~s}$. Afterwards, the fluctuations on the liquid velocity are stabilized and the time-averaged results over the last 120s are obtained.

A
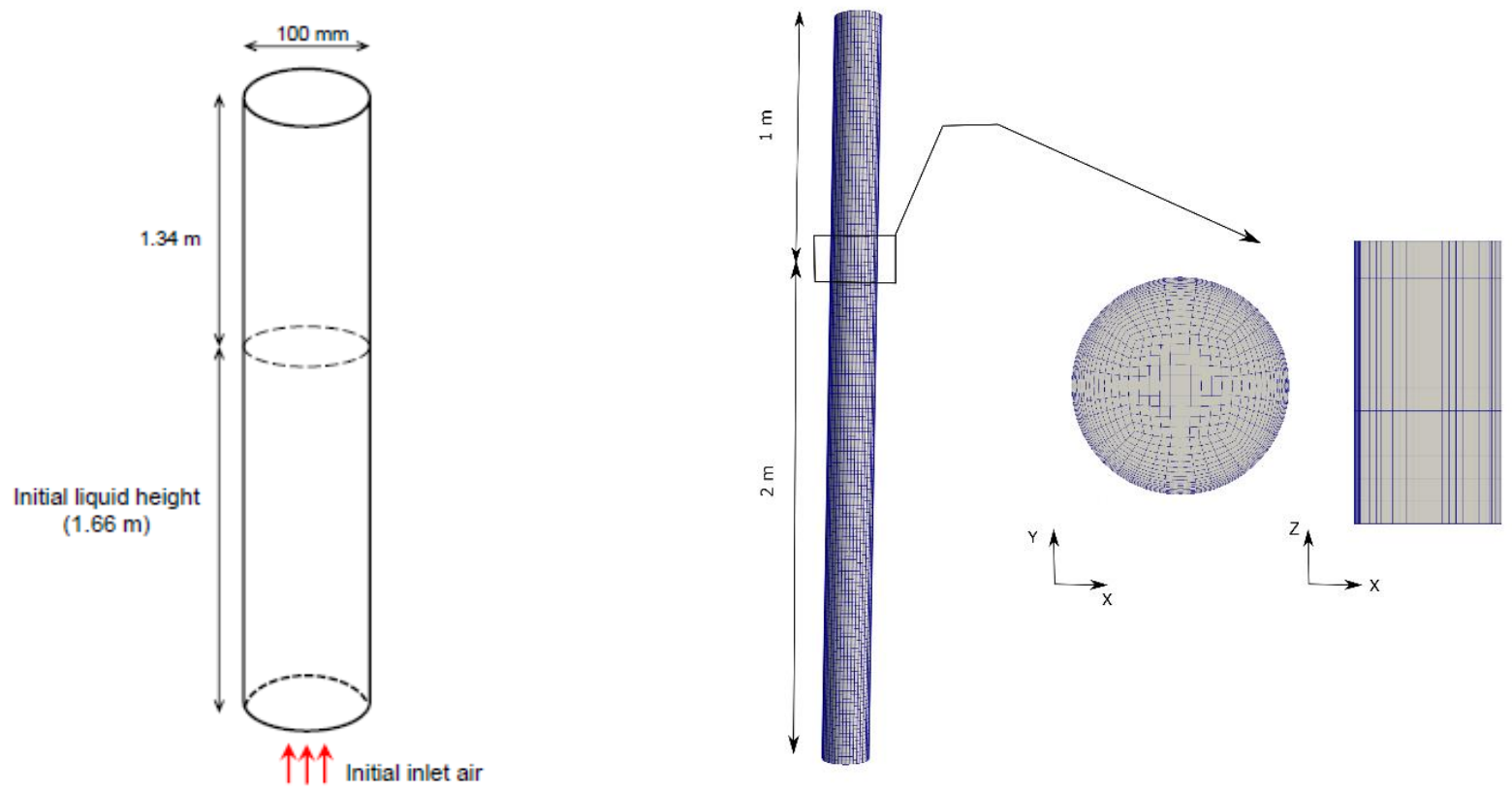

Fig. 1: Schematic view of the BCR geometry, $\mathbf{U}_{G}=0.06 \mathrm{~m} / \mathrm{s}$. (A), Mesh geometry of the bubble column in the vertical and radial direction (B). 
Table 1: Boundary conditions for inlet, outlet and wall patches.

\begin{tabular}{|c|c|c|c|}
\hline Variable & Inlet & Outlet & Wall \\
\hline Gas volume fraction & Dirichlet & Neumann & Slip \\
\hline Gas Velocity & Dirichlet & Neumann & No-slip \\
\hline Liquid Velocity & Dirichlet & Neumann & Neumann \\
\hline Pressure & Neumann & Dirichlet & \\
\hline
\end{tabular}

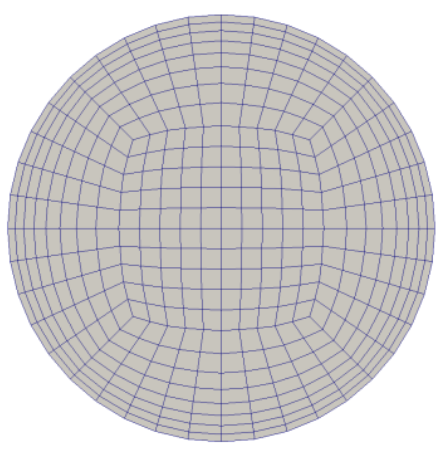

A

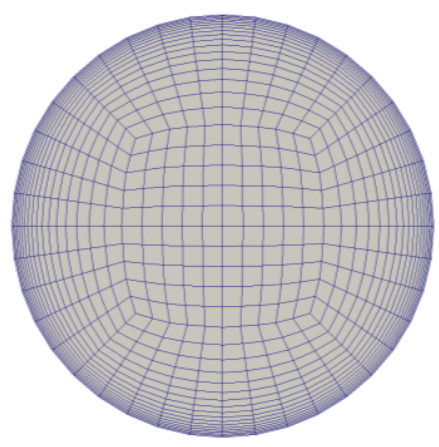

B

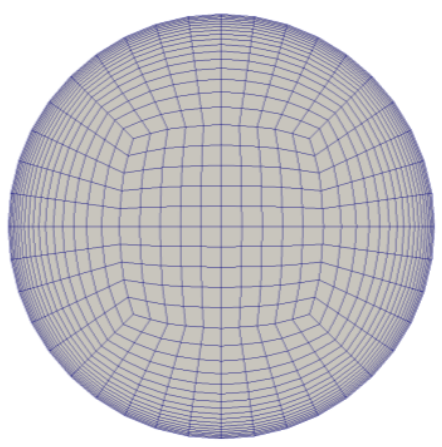

$\mathrm{C}$

Fig. 2: Schematic of three different meshes used for the gas sparger (A: 79348 elements, B: 123700 elements, and C: 136372 elements).
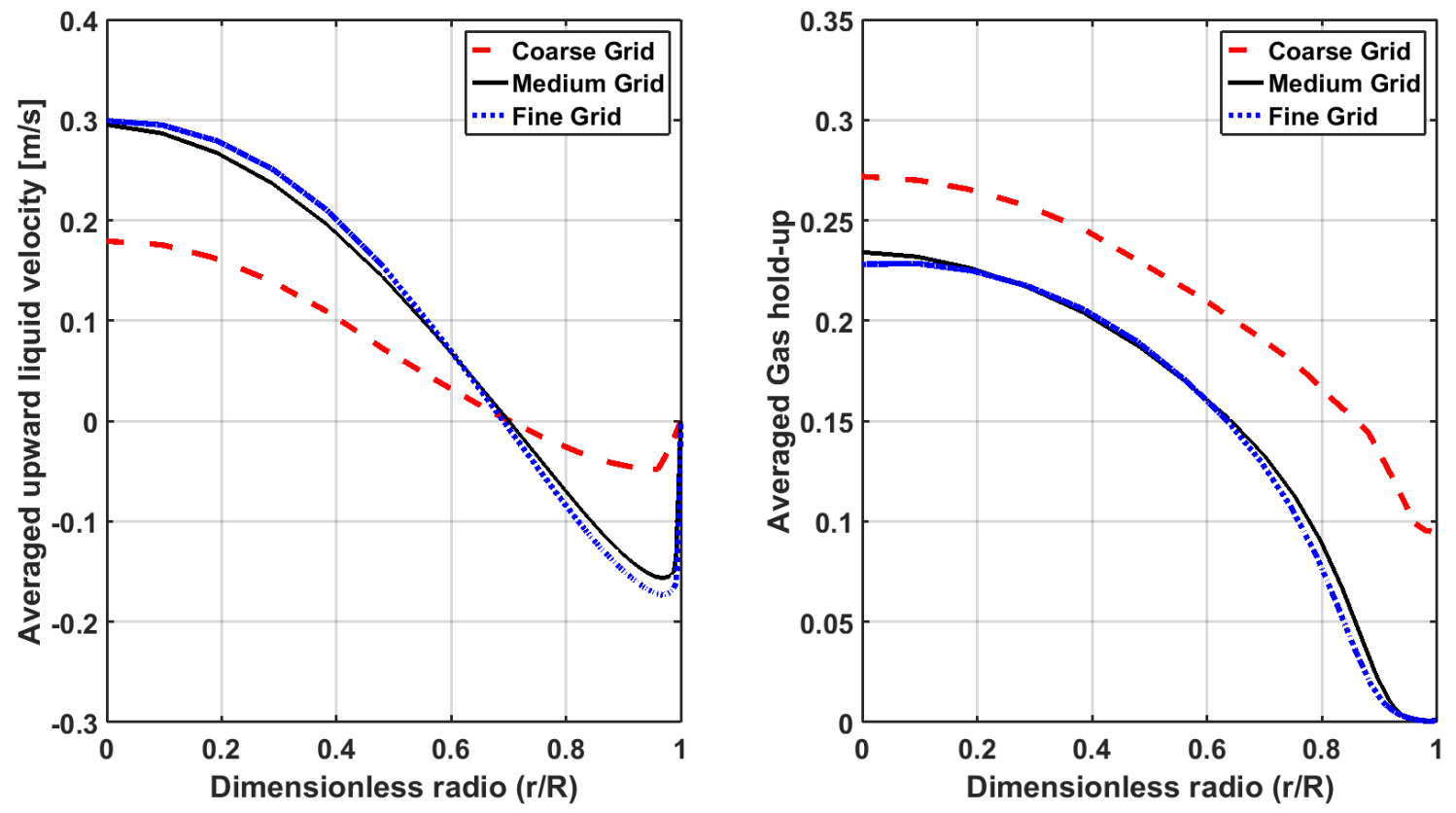

Fig. 3: Comparaison of the gas hod-up and liquid velocity for three diffrent meshes.

\section{Results and discussions}

In this reseach, firstly, local flow structure for gas-water system for intial superficial gas velocity $\mathbf{U}_{G}=0.06 \mathrm{~m} / \mathrm{s}$ is studied. Afterward, the role of different liquid system on flow pattern on BCR is examined. Figure 4 compares the CFD results and experimental measurements of the local hold-up and liquid velocity of Camarasa et al. [5]. Their experiments 
were conducted in the column having the same cross section diameter (100 mm)as this numerical study, using the same gas distributor and at a superficial velocity of $0.06 \mathrm{~m} \mathrm{~s}^{-1}$.

It was realized that there is good agreement between the experimental data and CFD preditions using a mean bubble size of $4 \mathrm{~mm}$, however, the CFD model underestimates the gas holdup at the zone near the wall. The similar behaviour was observed when comparing the simulations performed with $2 \mathrm{~mm}$ and $5 \mathrm{~mm}$ bubble diameters.

It is well known that the aqueous alcohol systems reduce the mean bubble size and the bubbly flow is characterized by the presence of uniform bubbles. From the experimental work [5], a mean size bubble of $2 \mathrm{~mm}$ is considered in this CFD model. Figure 5 displays a comparaison between the predicted time-averaged gas holup-up for pure water and water alcohol system for the $x y$-plane and $y z$-plane.At the superficial velocity $\mathbf{U}_{G}=0.06 \mathrm{~m} / \mathrm{s}$, the flow become unstable in air/water system and the transition regime appears as observed experimentally by Gourich et al. [6]. In the presence of the alcohol the flow become stable.As it is expected, the addition of propanol to air/water system leads to an increase of gas hold-up toward the top of the column.

The height of free surface increases from almost $1.857 \mathrm{~m}$ for air/water system to almost $2 \mathrm{~m}$ for air/water-alcohol solution. Since the coalescence is inhibited, the overall gas hold-up increases due to the presence of small bubbles in the column. Similar results were obtained in $[5,6,8]$. A comparaison between the simulated liquid velocity profiles for both air/water and air/water alcohol systems is displayed in Fig. 6. It is illusterated that upward liquid velocity for air/water system has small difference with air/water-alcohol solution.
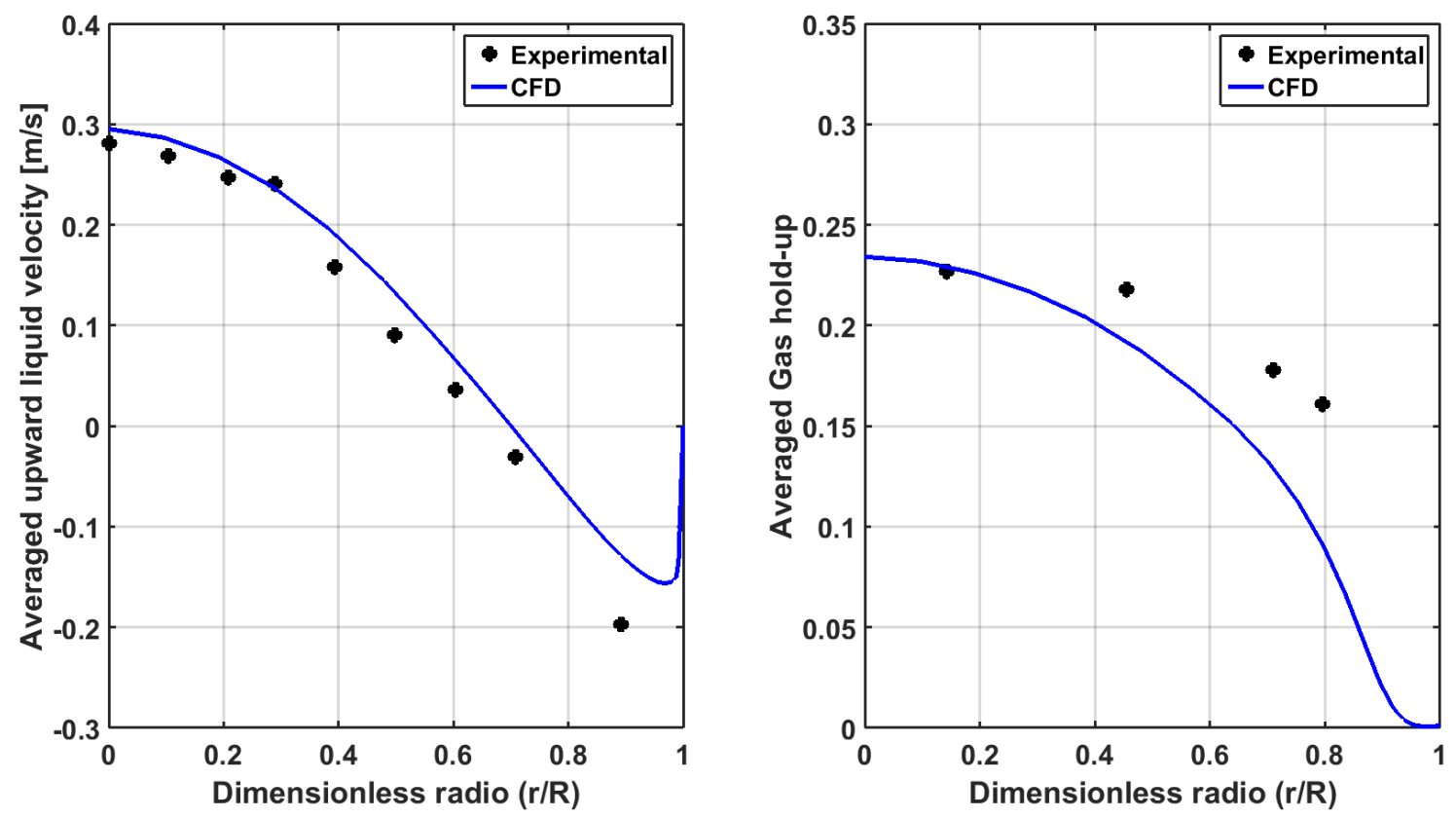

Fig. 4: Comparaison of CFD results and experimental data. 

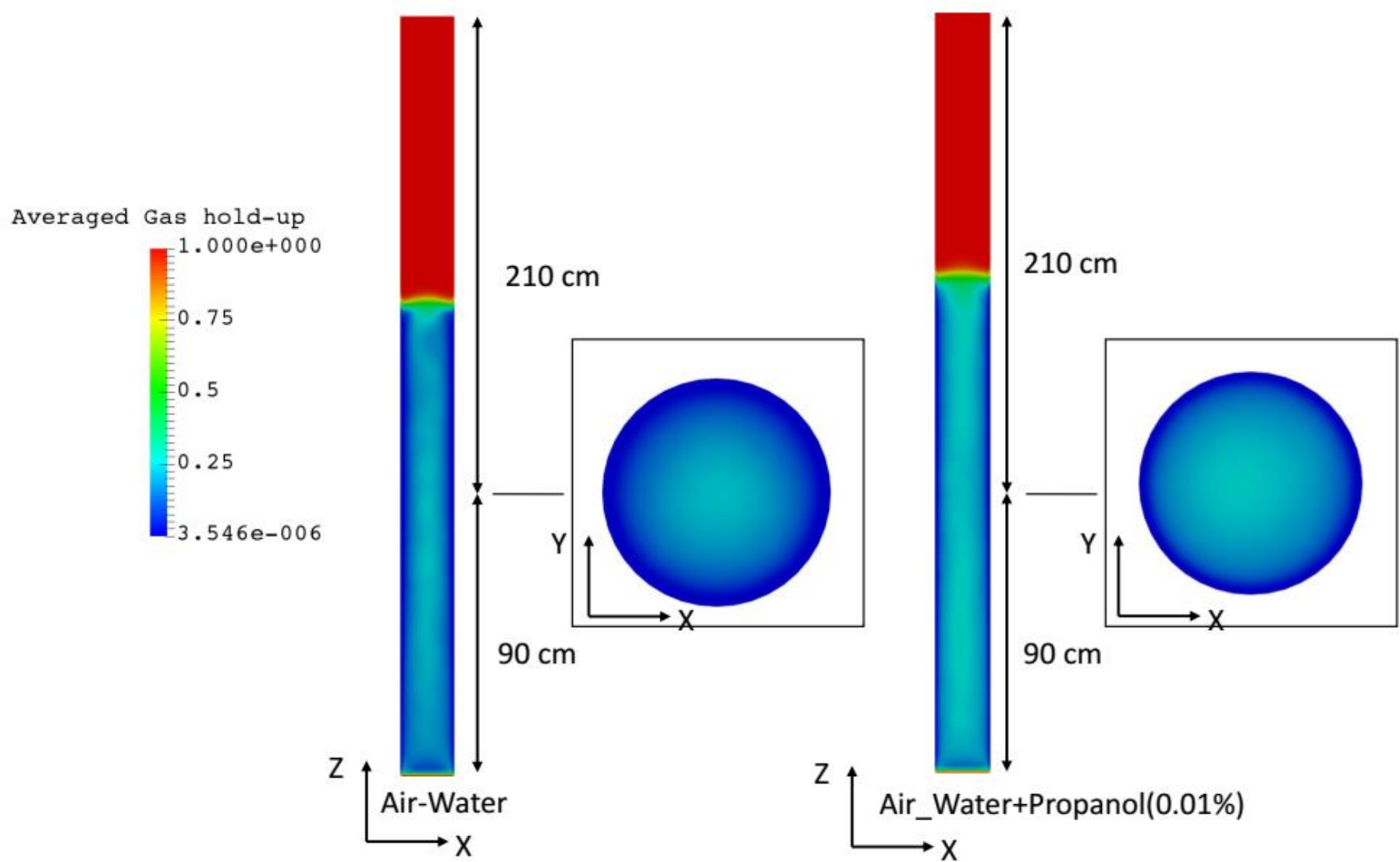

$90 \mathrm{~cm}$

Fig. 5: Averaged gas hold-up contour plot for Air-Water system in Z-X and Y-X in BCR.
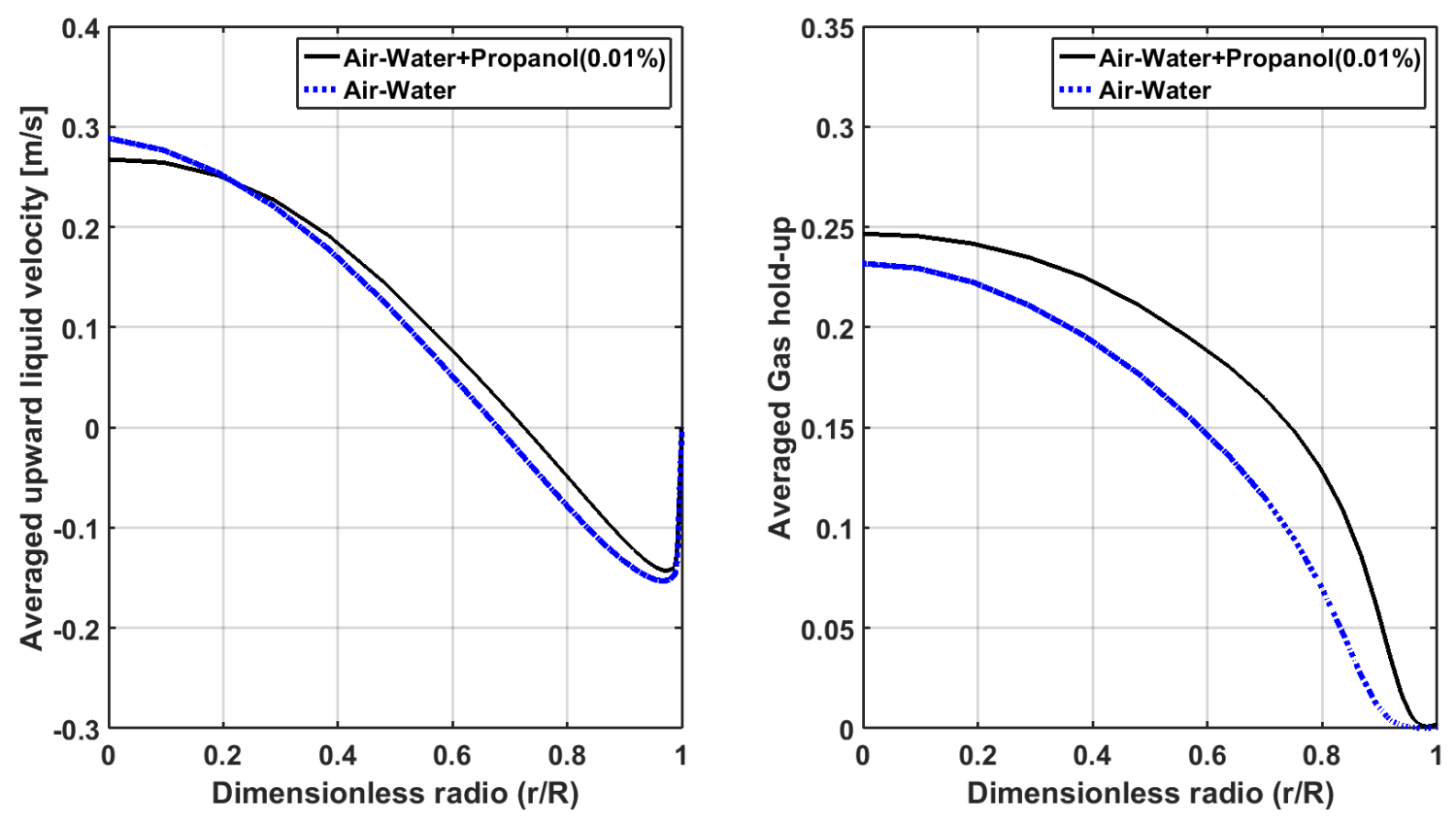

Fig. 6: Comparision of Air_Water with Air_Water+Propanol(0.01\%). 


\section{Conclusion}

In this perspective, numerical study of gas-liquid dispersion as well as the effect of alcohol addition (0.01\% propanol addition) on gas hold-up, liquid circulation velocity and gas velocity in flow structure within BCR are examined. in this research, a multiphase Euler- Euler method with mean bubble size and standard $k-\varepsilon$ turbulent model are utilized. The accuracy of the CFD model was validated by exprimental data. As it is indicated numerically, the addition of propanol $(0.01 \%)$ to air/water system leads to an increase of gas hold-up toward the top of the column. The upward liquid velocity has small difference. Further numerical experiments for heterogeneous flow regime is under way and will be reported in a future work.

\section{Acknowledgements}

This study has been supported by the Spanish Ministerio de Economía y Competitividad under project DPI201675791-C2-1-P.

\section{References}

[1] Ch. Vial and Y. Stiriba, "Characterization of Bioreactors Using Computational Fluid Dynamics," CRC Press Taylor and Francis Group, pp. 121-164, 2013.

[2] R. Krishna, M. I. Urseanu, J. M. van Baten and J. Ellenberger, "Influence of scale on the hydrodynamics of bubble columns operating in the churn-turbulent regime: experiments vs. Eulerian simulations," Chemical Engineering Science, vol. 54, No 21, pp. 4903-4911, 1999.

[3] B. Selma, R. Bannari and P. Proulx, "A full integration of a dispersion and interface closures in the standard model of turbulence," Chemical Engineering Science, vol. 65, pp. 5417-5428, 2010.

[4] V. V. Buwa and V. V. Ranade, "Dynamics of gas-liquid flow in a rectangular bubble column: experiments and single/muli-group CFD simulation," Chemical Engineering Science, vol. 57, pp. 4715-4736, 2002.

[5] E. Camarasa, Ch. Vial, S. Poncin, G. Wild, N. Midoux and J. Bouillard, "Influence of coalescence behavior of the liquid and of gas sparging on hydrodynamics and bubble characteristics in a bubble column," Chemical Engineering and Processing, vol. 38, pp. 329-344, 1999.

[6] B. Gourich, Ch. Vial, N. El Azher, M. Soulami Belhaj and M. Ziyad, "Influence of hydrodynamics and probe response on oxygen mass transfer measurements in a high aspect ratio bubble column reactor: Effect of the coalescence behavior of the liquid phase,"Biochemical Engineering Journal, vol. 39, No 1, pp. 1-14, 2008.

[7] G. Besagni, F. Inzoli, G. De Guido and L. A. Pellegrini, "Experimental investigation on the influence of ethanol on bubble column hydrodynamics," Chemical Engineering Research and Design, vol. 112, pp. 1-15, 2016.

[8] D. D. McClure, H. Nuris, J. M. Kavanagh, D. Fletcher and G. W. Barton, "Towards a CFD model of bubble columns containing significant levels," Chemical Engineering Science, vol. 127, pp. 189-201, 2015.

[9] B. Giorgio, F. Inzoli, T. Ziegenhein and D. Lucas, "Computatrional Fluid-Dynamic modeling of the pseudohomogeneous flow regime in large-scale bubble columns", Chemical Engineering Science, vol. 160, pp. 144-160, 2017.

[10] R. Clift, J. R. Grace and M. E. Weber, "Bubbles, drops and Particles," Academic Press, New York, 1978.

[11] OpenFOAM Ltd., United Kingdom (2012), OpenFOAM 2.1.1 user's guide. 